\title{
The management of hemiplegic shoulder pain in stroke subjects undergoing pulsed radiofrequency treatment of the suprascapular and axillary nerves: a pilot study
}

\author{
Congxian Yang ${ }^{1}$, Han $\mathrm{Xu}^{2}$, Rui Wang ${ }^{3}$, Yanhong Liu ${ }^{4}$, Shengtao Wang ${ }^{1}$ \\ ${ }^{1}$ Analgesia Department of Shandong Provincial Hospital Affiliated to the Shandong First Medical University, Jinan, China; ${ }^{2}$ Imaging Department \\ of Shandong Provincial Hospital Affiliated to the Shandong First Medical University, Jinan, China; ${ }^{3}$ Analgesia Department of Qingdao Municipal \\ Hospital, Qingdao, China; ${ }^{4}$ Yidu Central Hospital, Weifang, China \\ Contributions: (I) Conception and design: S Wang; (II) Administrative support: C Yang; (III) Provision of study materials or patients: C Yang; (IV) \\ Collection and assembly of data: H Xu, Y Liu; (V) Data analysis and interpretation: R Wang; (VI) Manuscript writing: All authors; (VII) Final \\ approval of manuscript: All authors. \\ Correspondence to: Shengtao Wang. Shandong Provincial Hospital Affiliated to the Shandong First Medical University, \#324, Jingwu Weiqi Road, \\ Jinan, China. Email: wshengt@126.com.
}

Background: Our trial aims to provide evidence for pain management and rehabilitation in patients with hemiplegic shoulder pain (HSP). HSP is one of the most common pains and disabilities occurring after a stroke. With accumulating evidence, the management of the suprascapular nerve (SSN) or axillary nerve (AN) might effectively relieve the pain and disability associated with HSP. However, no study has compared the effects of pulsed radiofrequency and nerve block of SSN and AN.

Methods: Twenty patients with chronic stroke (over one year from onset) and HSP [visual analog scale (VAS) for pain $\geq 30 \mathrm{~mm}$ ] randomly underwent ultrasound-guided SSN and AN pulsed radiofrequency or nerve block treatment. All patients were evaluated before treatment (T0) and at 4 (T1) and 16 (T2) weeks of follow-up. The primary outcome was the VAS score. Secondary outcomes were the Modified Ashworth Scale (MAS) score, passive shoulder range of motion (PROM), Disability Assessment Scale (DAS) score, and EuroQol-5 dimension questionnaire (EQ-5D).

Results: Significant improvements in the VAS score were observed in both groups at T1 and T2. However, a significant difference was not observed between the two groups ( $\mathrm{T} 1: \mathrm{P}=0.43$; $\mathrm{T} 2$ : $\mathrm{P}=0.23$ ). No statistically significant differences were observed in the MAS score between the two groups at $\mathrm{T} 1(\mathrm{P}=0.06)$ and $\mathrm{T} 2$ $(\mathrm{P}=0.07)$. In the PROM of shoulder abduction and external rotation, statistically, significant differences were observed between the two groups at T1 $\left(\mathrm{P}=0.02^{*}, \& \mathrm{P}=0.04^{*}\right)$ and $\mathrm{T} 2\left(\mathrm{P}=0.02^{*}, \& \mathrm{P}=0.00^{*}\right)$. Statistically significant differences in shoulder flexion and extension were not observed between the two groups at $\mathrm{T} 1$ $(\mathrm{P}=0.23, \& \mathrm{P}=0.35)$ and $\mathrm{T} 2(\mathrm{P}=0.14, \& \mathrm{P}=0.14)$. Statistically significant differences in the DAS score were not observed between the 2 groups at $\mathrm{T} 1(\mathrm{P}=0.51, \& \mathrm{P}=0.33, \& \mathrm{P}=0.36, \& \mathrm{P}=0.75)$ and $\mathrm{T} 2(\mathrm{P}=0.12, \&$ $\mathrm{P}=0.54, \& \mathrm{P}=0.41, \& \mathrm{P}=0.86)$. No statistically significant differences in the EQ-5D responses were observed between the two groups at $\mathrm{T} 1(\mathrm{P}=0.42)$ and $\mathrm{T} 2(\mathrm{P}=0.11)$.

Conclusions: Pulsed radiofrequency of SSN and AN achieves similar therapeutic effects to the nerve block. Pulsed radiofrequency modulation is superior to nerve block in improving the PROM of shoulder abduction and external rotation.

Keywords: hemiplegic shoulder pain (HSP); pulsed radiofrequency; suprascapular nerve (SSN); axillary nerve (AN); analgesia

Submitted Jul 04, 2020. Accepted for publication Sep 08, 2020.

doi: 10.21037/apm-20-1618

View this article at: http://dx.doi.org/10.21037/apm-20-1618 


\section{Introduction}

Hemiplegic shoulder pain (HSP) is a well-known type of chronic pain and disability experienced by patients after stroke (1). Within one year after the onset of stroke, the proportion of patients with HSP is as high as $30 \%$, a majority with moderate to severe pain (2). HSP not only causes severe pain but also causes depression, shoulder dysfunction, and substantially reduces the quality of life (3). Its pathophysiological mechanisms include impaired motor function (muscle tone changes), tendonitis, capsulitis, and neuropathic pain $(4,5)$. Many treatment methods have been described, including physical therapy, analgesic medications, transcutaneous electrical nerve stimulation, intra-articular injection, intramuscular botulinum injection and so on (5). However, evidence about the therapeutic effects is limited.

Pulsed radiofrequency is a neuro-modulatory technique that uses brief, high-voltage current bursts to modulate the nociceptive nerve function (6-8). Unlike conventional continuous radiofrequency treatment, pulsed radiofrequency applied high-voltage radiofrequency current bursts, followed by a relatively long "silent phase" to wash out the generated heat, so it exerts more reversible and less destructive effects (8). Pulsed radiofrequency might produce sustained inhibition of nociceptive or neuropathic input by altering synaptic transmission, propagation $(6,9,10)$. In vitro research also demonstrated that pulsed radiofrequency could affect cell morphology and some substrates, including c-fos, to activate neurons (6). This neuro-modulatory treatment can be used to the central nervous system (dorsal root ganglion and Gasserian ganglion) and the peripheral nervous system (10). It is effective for radicular pain from spinal diseases, postherpetic neuralgia, occipital neuralgia and so on (8).

Many targeted therapeutic procedures aimed at discrete peri-shoulder nerves, SSN (11-13). Most of the researches, seems not quite satisfactory $(12,14)$. Since with accumulating evidence, pain in the anterior or lateral aspect of the shoulder, the subacromial bursa and the area around the long head of the biceps tendon is related to the articular branch originating from the axillary nerve (AN) $(15,16)$. There was also evidence that the AN combined with SSN can be used as regional anesthesia for arthroscopic operation. Thus we added the AN to the suprascapular nerve (17).

Since pulsed radiofrequency could achieve prolonged analgesia and neuromodulatory effects, we postulated that pulsed radiofrequency is better than nerve block in consideration of pain relief, and might provide more benefits to spasm reduction and rehabilitation assistance. Therefore, we conducted this randomized pilot study. We present the following article in accordance with the CONSORT reporting checklist (available at http://dx.doi. org/10.21037/apm-20-1618).

\section{Methods}

This single-center pilot study analyzed data from 20 patients with chronic stroke and HSP who visited our analgesia department from September 2017 to May 2018.

The inclusion criteria were a diagnosed case of HSP, age $\geq 18$ and $\leq 80$ years, first-ever unilateral stroke, visual analog scale (VAS) $\geq 30 \mathrm{~mm}$, time since stroke $\geq 1$ year, and time since last local intervention treatment $>6$ months.

The exclusion criteria are participation in other trials, change in pain medication during the follow-up period, neuroleptic or surgical procedures for upper limb spasticity, other conditions in the affected shoulder (rotator cuff disorders, frozen shoulder, thoracic outlet syndrome, osteoarthritis, bursitis, recent trauma, bone fracture, joint replacement), and the patient is unable to complete the evaluations. There were no important changes to methods after trial commencement.

The 20 patients were randomly divided into two groups following the random number table: pulsed radiofrequency group, Group P ( $\mathrm{n}=12)$ and nerve block group, Group B $(\mathrm{n}=8)$. All patients were inpatients and provided written informed consent. This study was approved by Biomedical Research Ethic Committee of Shandong Provincial Hospital (No. SWYX2017-676). Patients did not take part in any rehabilitation program during the follow-up period. All procedures performed in this study involving human participants were in accordance with the Declaration of Helsinki (as revised in 2013).

\section{Treatment procedures}

The same physician performs all procedures.

\section{Group P (18)}

All patients were in the sitting position. First, local anesthesia was administered with $2 \mathrm{~mL}$ of $1 \%$ lidocaine to alleviate the pain of puncture. Under ultrasound guidance, the anatomical landmarks were determined (the suprascapular notch in the supraspinous fossa, Figure 1). A 22 -gauge, $100 \mathrm{~mm}, 5 \mathrm{~mm}$ active-tip radiofrequency needle 

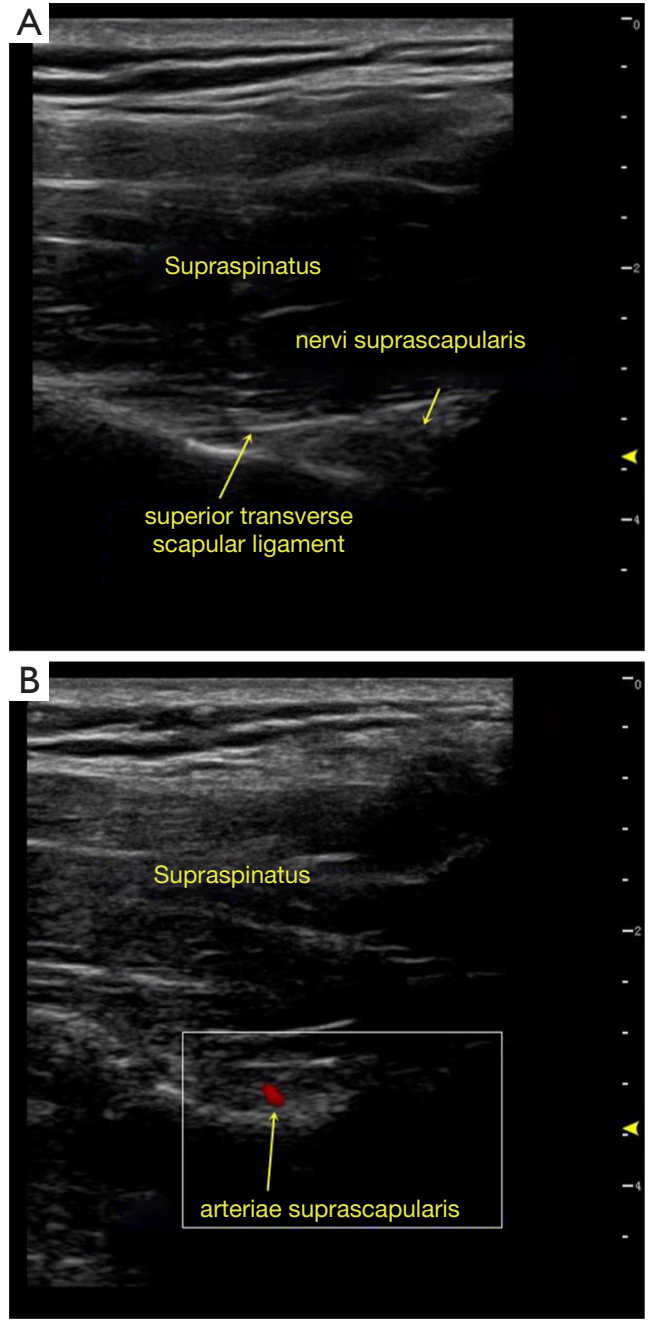

Figure 1 Ultrasonography of the suprascapular nerve. (A) The patient was in the sitting position, put the probe almost vertically above the Supraspinatus fossa, gently move the probe, when the bony structure was interrupted, such ultrasonography can be obtained; (B) confirm the arteriae suprascapularis by Color Doppler Mode. The suprascapular nerve is beside the arteriae suprascapularis.

was guided near the arteriae suprascapularis. Following the elicitation of a paresthesia response in the shoulder region to a $50 \mathrm{HZ}, 1 \mathrm{~ms}, 0.3 \mathrm{~V}$ sensory stimulus and appropriate muscular response to a $2 \mathrm{HZ}, 1 \mathrm{~ms}, 0.3 \mathrm{~V}$ stimulus, pulsed radiofrequency treatment was applied at $42{ }^{\circ} \mathrm{C}, 600 \mathrm{~s}$, $100 \mathrm{~V}, 10 \mathrm{~ms}$, and $1 \mathrm{~Hz}$ (the radiofrequency equipment is Baylis PMG-230, Canada). Then, under ultrasound guidance, the needle was inserted in the quadrilateral foramen near the arteriae circumflexa humeri posterior (11).
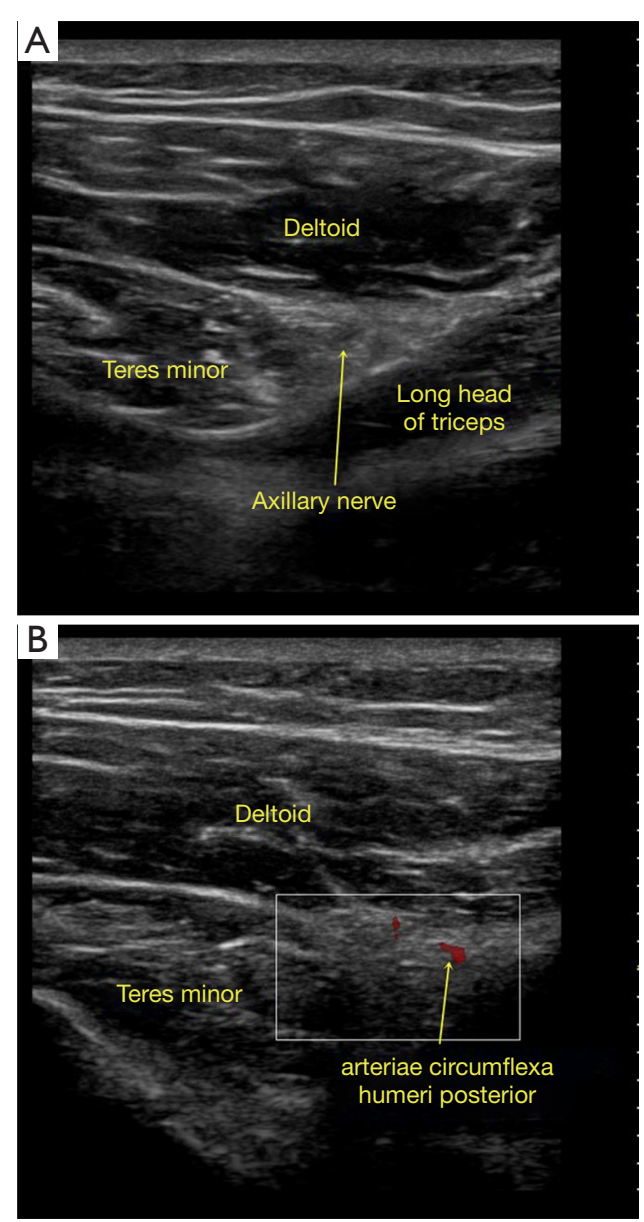

Figure 2 Ultrasonography of the axillary nerve. (A) The patient was in the sitting position, put the probe behind the shoulder, gently move the probe, distinguish the triceps brachii and teres minor; (B) confirm the arteriae circumflexa humeri posterior by Color Doppler Mode. The axillary nerve is beside the arteriae circumflexa humeri posterior.

Similar parameters of pulsed radiofrequency were applied (Figure 2).

\section{Group B}

All patients were in the sitting position. First, local anesthesia was administered with $2 \mathrm{~mL}$ of $1 \%$ lidocaine. Under ultrasound guidance, the anatomical landmarks were found (the suprascapular notch in the supraspinous fossa). A 7 -gauge, $80 \mathrm{~mm}$ needle was guided near the arteriae suprascapularis, and $8 \mathrm{~mL}$ of a mixture of $5 \mathrm{~mL}$ of $2 \%$ lidocaine (Shiyao Pharmaceutical Co., Ltd., China), 
Table 1 The demographic data of the patients with HSP

\begin{tabular}{lccc}
\hline Characteristics & Group P $(\mathrm{n}=12)$ & Group B $(\mathrm{n}=8)$ & $\mathrm{P}$ value \\
\hline Sex (female/male) & $5 / 7$ & $5 / 3$ & 0.650 \\
Duration(m), mean (SD) & $24.83(8.70)$ & $20.63(4.81)$ & 0.231 \\
VAS (0-100 mm), mean (SD) & $65.83(10.84)$ & $63.75(13.03)$ & 0.702 \\
EQ-5D (0-100 mm), mean (SD) & $53.00(8.18)$ & $57.25(4.68)$ & 0.202 \\
\hline
\end{tabular}

HSP, hemiplegic shoulder pain; VAS, visual analog scale; SD, standard deviation; EQ-5D, EuroQol-5 dimension questionnaire.

$7 \mathrm{mg}$ of Diprospan ${ }^{\circledR}$ (Schering-Plough Labo N.V., Belgium) and $10 \mathrm{~mL}$ of normal saline were administered. Then, under ultrasound guidance, the needle was inserted in the quadrilateral foramen near the arteria circumflexa humeri posterior (11). Another $8 \mathrm{~mL}$ of the mixture was then applied.

\section{Evaluation procedure}

All patients were evaluated before treatment (T0) and after four weeks (T1) and 16 weeks (T2). All data were collected and analyzed by the same professional who knew nothing about the details of the study.

\section{Primary outcome}

The primary outcome was the VAS score for pain, in which a $100 \mathrm{~mm}$ horizontal line is drawn on paper: one end of the line is 0 , indicating no pain; the other end is 100 , indicating the severest pain imaginable; and the middle part indicates different degrees of pain (19).

\section{Secondary outcomes}

Secondary outcomes are the Modified Ashworth Scale (MAS) score, the passive shoulder range of motion (PROM), the Disability Assessment Scale (DAS) score, and the EuroQol-5 dimension questionnaire (EQ-5D).

The MAS is used to measure shoulder abductor muscle tone. This 6-point scale grades resistance to rapid passive stretch from 0 (no increase in muscle tone) to 5 (the joint is rigid) $(20,21)$. Shoulder flexion, extension, abduction, and external rotation were measured using a handheld goniometer. The sensitivity of the measurement was arbitrarily set to $5^{\circ}(22)$. The DAS was used to evaluate the extent of functional impairment in the domains of patient hygiene, dressing, limb position, and pain as follows: 0 , no disability; 1 , mild disability; 2, moderate disability; and 3, severe disability $(23,24)$. Quality of life was assessed using the EQ-5D on a visual scale from 0 (the worst imaginable health state) to 100 ( the best imaginable health state) points (25). There were no changes to trial outcomes after the trial commenced.

\section{Statistical analysis}

Statistical analyses were performed using SPSS software, version 17.0 (IBM Corporation, Armonk, NY, USA). The VAS score, PROM, and EQ-5D responses were first subjected to a test of the homogeneity of variance. If the homogeneity of variance was confirmed, an independent sample t-test was selected for the comparison between the two groups. If the heterogeneity of variance was observed, the Wilcoxon rank-sum test was selected, and $\mathrm{P}<0.05$ was considered statistically significant. The MAS and DAS scores were compared using the Wilcoxon rank-sum test, and $\mathrm{P}<0.05$ was considered statistically significant. Differences in the VAS score, PROM, and EQ-5D between $\mathrm{T} 1$ and $\mathrm{T} 0$ and between $\mathrm{T} 2$ and $\mathrm{T} 0$ in the same group were compared using the paired sample $T$-test; $\mathrm{P}<0.05$ was considered statistically significant. MAS and DAS scores were compared between time points using the paired sample rank-sum test, and $\mathrm{P}<0.05$ was considered statistically significant.

\section{Results}

No adverse events occurred during the follow-up period. Table 1 presents the demographic and clinical characteristics of each group. Statistically, differences were not observed between the two groups.

\section{Primary outcome}

Significant improvements in the VAS score of Group P were observed at $\mathrm{T} 1$ and $\mathrm{T} 2$. The same results were obtained for group B. However, a significant difference in the VAS score was not observed between the two groups at $\mathrm{T} 1(\mathrm{P}=0.43)$ and $\mathrm{T} 2(\mathrm{P}=0.23)$ (Table 2). 


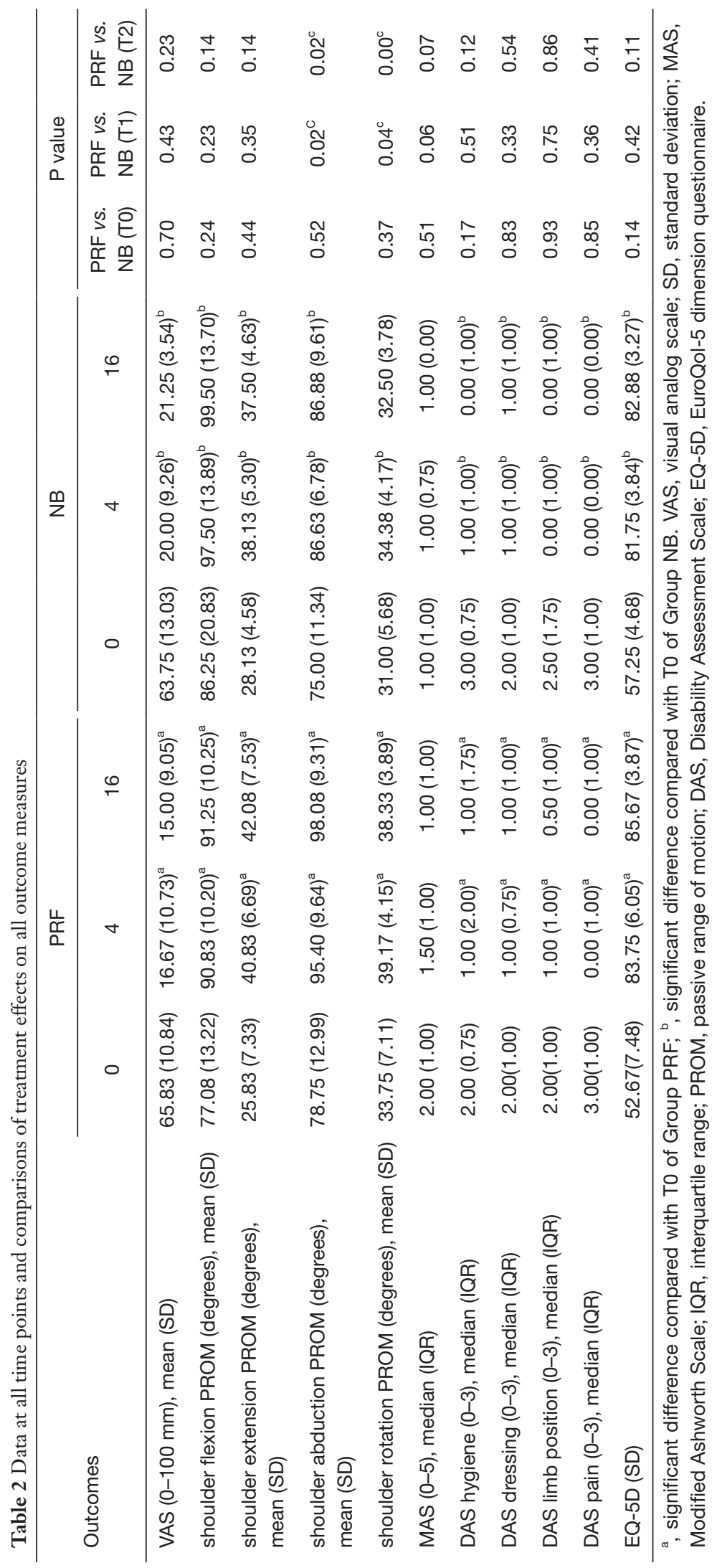




\section{Secondary outcomes (Table 2)}

No significant improvement in the MAS score was observed in both groups at T1 and T2. Statistically significant differences in the MAS score were not observed between the two groups at $\mathrm{T} 1(\mathrm{P}=0.06)$ and $\mathrm{T} 2(\mathrm{P}=0.07)$.

Significant improvements in the PROM of shoulder flexion, shoulder extension, and shoulder abduction were observed in both groups at T1 and T2. Significant improvements in external shoulder rotation were observed in group $\mathrm{P}$ at $\mathrm{T} 1$ and $\mathrm{T} 2$, but only at T1 in group $\mathrm{B}$. No significant improvements in the external rotation were observed in Group B at T2. A statistically significant difference was observed between the two groups in the PROM of shoulder abduction and external rotation at $\mathrm{T} 1\left(\mathrm{P}=0.02^{*}, \& \mathrm{P}=0.04^{*}\right)$ and $\mathrm{T} 2\left(\mathrm{P}=0.02^{*}, \& \mathrm{P}=0.00^{*}\right)$. Statistically significant differences in shoulder flexion and extension were not observed between the two groups at $\mathrm{T} 1$ $(\mathrm{P}=0.23, \& \mathrm{P}=0.35)$ and $\mathrm{T} 2(\mathrm{P}=0.14, \& \mathrm{P}=0.14)$.

Significant improvements in the DAS score for hygiene, dressing, pain, and limb position were observed in both groups at $\mathrm{T} 1$ and $\mathrm{T} 2$. No statistically significant differences in the DAS score were observed between the 2 groups at T1 $(\mathrm{P}=0.51, \& \mathrm{P}=0.33, \& \mathrm{P}=0.36, \& \mathrm{P}=0.75)$ and $\mathrm{T} 2(\mathrm{P}=0.12$, \& $\mathrm{P}=0.54, \& \mathrm{P}=0.41, \& \mathrm{P}=0.86$ ).

Significant improvements in the EQ-5D were observed in both groups at T1 and T2. Statistically significant differences in the EQ-5D were not observed between the two groups at $\mathrm{T} 1(\mathrm{P}=0.42)$ and $\mathrm{T} 2(\mathrm{P}=0.11)$.

\section{Discussion}

In the present study, we examine the effectiveness of pulsed radiofrequency of SSN and AN in the treatment of HSP by using a randomized controlled trial. We supposed that pulsed radiofrequency could provide longer-lasting or better analgesic effect than nerve block. However, it appears that both therapies help reduce pain, but none statistically significant difference exists between the two groups at 4 and 16 weeks in the analgesic effect. The three shoulder PROM directions improved in both groups by 16 weeks, except for external rotation during the followup. Compared with-nerve block, the pulsed radiofrequency treatment appeared to achieve more significantly improvements in the abduction and external rotation.

HSP is a frequent sequela after stroke with a high incidence, which increases the duration of hospitalization and substantially impairs the rehabilitation process. The mechanism by which a rigid, painful shoulder occurs after stroke has not been conclusively determined and still is controversial. Many treatment methods have been described, ranging from physical therapy to articular infiltration. However, evidence from therapeutic procedures is limited. Recently, more targeted therapeutic procedures might be considered for discrete peri-shoulder nerves, SSN and AN (11-13), due to the SSN and AN dominating most of the soft tissues of the shoulder region. Therefore, we performed this study to determine whether the treatment of SSN and AN effectively alleviates the local pain of the shoulder joint.

The suprascapular nerve (SSN), a branch of the superior trunk of the brachial plexus (C5 and C6), innervates the supraspinatus and infraspinatus muscles and provides $70 \%$ of sensory innervations to the shoulder joint $(26,27)$. The articular branch of SSN innervates the coracoclavicular ligaments, the coracohumeral ligament, the subacromial bursa, and the posterior parts of the shoulder capsule (27). In patients with HSP, the afferent fibers of the SSN can become entrapped by injured tissues or sensitized after longterm unresolved pain (28). However, these SSN therapies alone do not entirely solve the problem of shoulder pain and limited mobility in patients with HSP (29). With accumulating evidence, pain in the anterior or lateral aspect of the shoulder is postulated to originate from the SSN, might be related to the articular branch originating from the AN and innervate the subacromial bursa and the area around the long head of the biceps tendon $(15,16)$. In the present study, the treatment targeted the SSN combined with the AN to relieve shoulder pain and improve function and movement.

Pulsed radiofrequency, a neuro-modulatory treatment, in which short bursts of high-voltage radiofrequency energy are applied to nervous tissue, appeared about 20 years ago as an alternative technique devoid of nerve injury, a subsequent side effect of thermal lesions created by continuous radiofrequency (6). It can be used to the central nervous system (dorsal root ganglia and Gasserian ganglion) and the peripheral nervous system (10). For the peripheral nervous system, pulsed radiofrequency of the selective nerve can impede nociceptive input, activate c-fos protein expression, induce dramatic metabolic and gene changes without morphologic cell changes at sub-cytotoxic levels that might (30). Since pulsed radiofrequency provides neuro-modulatory function, we suppose it might provide a longer or better analgesic effect, and could modulate the high muscle tension. However, it seems our result does not 
substantiate this inference.

For HSP patients, mechanisms of pain include not only nociceptive pain but also neuropathic pain and central sensitization $(4,28)$. The formation of neuropathic pain needs long-lasting peripheral nociceptive afferents. The nociceptive components might be caused by entrapment of the nervous system, tendinitis, bursitis, or arthritis. Injection of the local anesthetics can inhibit the nociceptive afferent and relieve the spasm of the surrounding tissues for several hours. The role of glucocorticoids relates to its anti-inflammatory action. Besides, the injection of mixed fluid might produce "liquid dilation." Pulsed radiofrequency of the selective nerve can impede nociceptive input, activate $\mathrm{c}$-fos protein expression, induce dramatic metabolic and gene changes without morphologic cell changes at sub-cytotoxic levels that might (30). Since pulsed radiofrequency provides neuro-modulatory function, we suppose it might alleviate both nociceptive and neuropathic components, and might provide a longer or better analgesic effect. However, it seems our result does not substantiate this inference. In our study, both therapies show pain reduction, with no significant difference between the two groups.

For HSP patients, high muscle tension, and dysfunction aggravate and worsen each other. Higuchi demonstrated that pulsed radiofrequency increased c-Fos immunoreactivity in the laminae I and II of the rat spinal cord (30). The expression of c-Fos, not specific for nociceptive pathways, is an indirect marker of neuronal activity as c-Fos is often expressed when neuron fire action potentials. Hamann demonstrated revealing an upregulation of activating transcription factor-3 (ATF-3), another marker of "cellular stress," lending further evidence to the definite biological effects of pulse radiofrequency (31). Its presence indicates the high electric burst activates nerve fibers. So we want to know about whether the high electric burst delivered by pulsed radiofrequency could modulate high muscle tension. However, according to the MAS scores, neither remedy could improve muscle tension. In our opinion, further research might consider pulsed radiofrequency to the dorsal root ganglia, spinal cord, or up to the dorsal horn of the spinal cord, because muscle spasms may be related to the central nervous system.

Both groups showed significant improvements in the DAS score and EQ-5D at the two-time points of followup, but the differences between the two groups were not significant. The pulsed radiofrequency group did not show superior outcomes to the nerve block group. Thus, both therapeutic methods effectively reduce joint disability and improve the quality of life for up to 16 weeks after treatment.

During the follow-up period, three directions of the shoulder PROM improved in both groups for 16 weeks, except for external rotation. Compared with patients in group B, the pulsed radiofrequency treatment appeared to achieve significantly more significant improvements in the abduction and external rotation. An assertion that the different treatments caused the differences is preliminary because the sample size was small. However, treatment of the SSN and AN might be proposed as a choice in stroke rehabilitation to facilitate shoulder mobilization and neuromotor techniques in patients with HSP.

To the best of our knowledge, this report is the first to compare the effects of an ultrasound-guided SSN and AN pulsed radiofrequency treatment with nerve block treatment in patients with chronic stroke experiencing HSP. However, some limitations should be considered when reviewing the results of this trial. First, a small sample size and limited follow-up were examined. The analgesic effect and improvement of function need further detailed research. Studies with a larger sample size are necessary to confirm the efficacy. Second, a control group treated with a placebo or other treatments (for example, intra-articular injection, botulinum toxin, or physical therapy) for HSP was not analyzed.

\section{Conclusions}

Our observations support using ultrasound-guided pulsed radiofrequency of the SSN and AN for HSP in patients with chronic stroke. Pulsed radiofrequency modulation and nerve block achieve similar therapeutic effects on analgesia and improvements in shoulder flexion and extension. Pulsed radiofrequency modulation is superior to nerve block in improving shoulder abduction and external rotation. Neither treatment improves muscle tension.

\section{Acknowledgments}

Funding: None.

\section{Footnote}

Reporting Checklist: The authors have completed the CONSORT reporting checklist. Available at http://dx.doi. org/10.21037/apm-20-1618 
Data Sharing Statement: Available at http://dx.doi. org/10.21037/apm-20-1618

Conflicts of Interest: All authors have completed the ICMJE uniform disclosure form (available at http://dx.doi. org/10.21037/apm-20-1618). The authors have no conflicts of interest to declare.

Ethical Statement: The authors are accountable for all aspects of the work in ensuring that questions related to the accuracy or integrity of any part of the work are appropriately investigated and resolved. This study was approved by Biomedical Research Ethic Committee of Shandong Provincial Hospital (No. SWYX2017-676). All procedures performed in this study involving human participants were in accordance with the Declaration of Helsinki (as revised in 2013). All patients were inpatients and provided written informed consent.

Open Access Statement: This is an Open Access article distributed in accordance with the Creative Commons Attribution-NonCommercial-NoDerivs 4.0 International License (CC BY-NC-ND 4.0), which permits the noncommercial replication and distribution of the article with the strict proviso that no changes or edits are made and the original work is properly cited (including links to both the formal publication through the relevant DOI and the license). See: https://creativecommons.org/licenses/by-nc-nd/4.0/.

\section{References}

1. Pundik S, McCabe J, Skelly M, et al. Association of spasticity and motor dysfunction in chronic stroke. Ann Phys Rehabil Med 2019;62:397-402.

2. Lindgren I, Jonsson AC, Norrving B, et al. Shoulder pain after stroke: a prospective population-based study. Stroke 2007;38:343-8.

3. Adey-Wakeling Z, Liu E, Crotty M, et al. Hemiplegic Shoulder Pain Reduces Quality of Life After Acute Stroke: A Prospective Population-Based Study. Am J Phys Med Rehabil 2016;95:758-63.

4. Zeilig G, Rivel M, Weingarden H, et al. Hemiplegic shoulder pain: evidence of a neuropathic origin. Pain 2013;154:263-71.

5. Coskun Benlidayi I, Basaran S. Hemiplegic shoulder pain: a common clinical consequence of stroke. Pract Neurol 2014;14:88-91.

6. Cahana A, Vutskits L, Muller D. Acute differential modulation of synaptic transmission and cell survival during exposure to pulsed and continuous radiofrequency energy. The Journal of Pain 2003;4:197-202.

7. Argyriou AA, Anastopoulou GG, Bruna J. Inconclusive evidence to support the use of minimally-invasive radiofrequency denervation against chronic low back pain. Ann Transl Med 2018;6:127.

8. Chang MC. Efficacy of Pulsed Radiofrequency Stimulation in Patients with Peripheral Neuropathic Pain: A Narrative Review. Pain Physician. 2018;21:E225-34.

9. Cahana A, Van Zundert J, Macrea L, et al. Pulsed radiofrequency: current clinical and biological literature available. Pain Med 2006;7:411-23.

10. Chua NH, Vissers KC, Sluijter ME. Pulsed radiofrequency treatment in interventional pain management: mechanisms and potential indications-a review. Acta Neurochir (Wien) 2011;153:763-71.

11. Gofeld M, Agur A. Peripheral Nerve Stimulation for Chronic Shoulder Pain: A Proof of Concept Anatomy Study. Neuromodulation 2018;21:284-9.

12. Picelli A, Lobba D, Vendramin P, et al. A retrospective case series of ultrasound-guided suprascapular nerve pulsed radiofrequency treatment for hemiplegic shoulder pain in patients with chronic stroke. J Pain Res 2018;11:1115-20.

13. Fernandes MR, Barbosa MA, Sousa ALL, et al. Suprascapular Nerve Block: Important Procedure in Clinical Practice. Rev Bras Anestesiol 2012;62:96-104.

14. Alanbay E, Aras B, Kesikburun S, et al. Effectiveness of Suprascapular Nerve Pulsed Radiofrequency Treatment for Hemiplegic Shoulder Pain: A Randomized-Controlled Trial. Pain Physician 2020;23:245-52.

15. Nasu H, Nimura A, Yamaguchi K, et al. Distribution of the axillary nerve to the subacromial bursa and the area around the long head of the biceps tendon. Knee Surg Sports Traumatol Arthrosc 2015;23:2651-7.

16. Flynn LS, Wright TW, King JJ. Quadrilateral space syndrome: a review. J Shoulder Elbow Surg 2018;27:950-6.

17. Nam YS, Jeong JJ, Han SH, et al. An anatomic and clinical study of the suprascapular and axillary nerve blocks for shoulder arthroscopy. J Shoulder Elbow Surg 2011;20:1061-8.

18. Dhir S, Sondekoppam RV, Sharma R, et al. A Comparison of Combined Suprascapular and Axillary Nerve Blocks to Interscalene Nerve Block for Analgesia in Arthroscopic Shoulder Surgery: An Equivalence Study. Reg Anesth Pain Med 2016;41:564-71.

19. Price CI, Curless RH, Rodgers H. Can stroke patients use 
visual analogue scales? Stroke 1999;30:1357-61.

20. Naghdi S, Ansari NN, Azarnia S, et al. Interrater reliability of the Modified Modified Ashworth Scale (MMAS) for patients with wrist flexor muscle spasticity. Physiother Theory Pract 2008;24:372-9.

21. Ansari NN, Naghdi S, Arab TK, et al. The interrater and intrarater reliability of the Modified Ashworth Scale in the assessment of muscle spasticity: limb and muscle group effect. NeuroRehabilitation 2008;23:231-7.

22. Picelli A, Bonazza S, Lobba D, et al. Suprascapular nerve block for the treatment of hemiplegic shoulder pain in patients with long-term chronic stroke: a pilot study. Neurol Sci 2017;38:1697-701.

23. Bustamante A, Garcia-Berrocoso T, Rodriguez N, et al. Ischemic stroke outcome: A review of the influence of post-stroke complications within the different scenarios of stroke care. Eur J Intern Med 2016;29:9-21.

24. Picelli A, Vallies G, Chemello E, et al. Influence of physician empathy on the outcome of botulinum toxin treatment for upper limb spasticity in patients with chronic stroke: A cohort study. J Rehabil Med 2017;49:410-5.

25. de Graaf JA, Kuijpers M, Visser-Meily J, et al. Validity of an enhanced EQ-5D-5L measure with an added cognitive dimension in patients with stroke. Clin Rehabil

Cite this article as: Yang C, Xu H, Wang R, Liu Y, Wang S. The management of hemiplegic shoulder pain in stroke subjects undergoing pulsed radiofrequency treatment of the suprascapular and axillary nerves: a pilot study. Ann Palliat Med 2020;9(5):3357-3365. doi: 10.21037/apm-20-1618
2020;34:545-50.

26. Agur AMR, Dalley AF. Grant's atlas of anatomy. 14th, editor: Wolters Kluwer; 2017.

27. Vorster W, Lange CP, Briet RJ, et al. The sensory branch distribution of the suprascapular nerve: an anatomic study. J Shoulder Elbow Surg 2008;17:500-2.

28. Borstad J, Woeste C. The role of sensitization in musculoskeletal shoulder pain. Braz J Phys Ther 2015;19:251-7.

29. Kay J, Memon M, Hu T, et al. Suprascapular Nerve Blockade for Postoperative Pain Control After Arthroscopic Shoulder Surgery: A Systematic Review and Meta-analysis. Orthop J Sports Med 2018;6:2325967118815859.

30. Higuchi Y, Nashold BS Jr, Sluijter M, et al. Exposure of the dorsal root ganglion in rats to pulsed radiofrequency currents activates dorsal horn lamina I and II neurons. Neurosurgery 2002;50:850-5; discussion 856.

31. Hamann W, Abou-Sherif S, Thompson S, et al. Pulsed radiofrequency applied to dorsal root ganglia causes a selective increase in ATF3 in small neurons. Eur J Pain 2006;10:171-6.

(English Language Editor: J. Chapnick) 